\title{
No-Touch Technique and a New Donor Adjuster for Descemet's Stripping Automated Endothelial Keratoplasty
}

\author{
Akira Kobayashi Hideaki Yokogawa Kazuhisa Sugiyama \\ Department of Ophthalmology, Kanazawa University Graduate School of \\ Medical Science, Kanazawa, Japan
}

\section{Key Words}

Descemet's stripping automated endothelial keratoplasty · No-touch technique · Donor adjuster

\begin{abstract}
Purpose: One difficulty with Descemet's stripping automated endothelial keratoplasty (DSAEK) is air management during surgery and donor endothelial lamella centering. We evaluated the no-touch technique for donor centering and the use of a newly developed DSAEK donor adjuster.
\end{abstract}

Methods: We evaluated the records of 12 consecutive patients (mean age 75.3 years) with bullous keratopathy who had undergone DSAEK. In all cases, the no-touch technique was attempted first. When the no-touch technique failed, a DSAEK donor adjuster with a 30gauge cannula resembling a curved reverse Sinskey hook was used for donor centering. The adjuster allows air injection during donor centering.

Results: The no-touch technique using simple corneal surface massage to center the graft was successful in 4 cases $(33.3 \%)$, while 4 cases required ocular tilting $(33.3 \%)$ in addition to corneal surface massage. The no-touch technique was ineffective in 4 cases (33.3\%), but the donor adjuster was used successfully and easily for these patients. Comparing the endothelial cell loss rate between the no-touch technique group and the donor adjuster group, there was no significant difference at 6 months.

Conclusions: The no-touch technique was useful for better control of DSAEK donor centering in most cases. When the no-touch technique was ineffective, the DSAEK donor adjuster was uniformly successful. 


\section{Introduction}

Over several years, new surgical techniques for the treatment of bullous keratopathy, which replace only the dysfunctional posterior portion of the cornea through a scleral pocket incision, have been reported [1,2]. These techniques completely eliminate central surface corneal incisions or sutures, maintain much of the cornea's structural integrity and induce minimal refractive change, suggesting distinct advantages over the standard penetrating keratoplasty. Furthermore, preparation of the donor tissue in endothelial keratoplasty has been made easier with the utilization of an automated microkeratome. The addition of this component to the surgical procedure has been popularized as Descemet's stripping automated endothelial keratoplasty (DSAEK) [3, 4]. Recently, we [5] and others [6, 7] eliminated Descemet stripping for non-Fuchs type bullous keratopathies and called the modified procedure non-DSAEK (nDSAEK) [5]. Both DSAEK and nDSAEK have proved to be quite effective for endothelial dysfunction of Asian eyes, with rapid visual recovery and minimal induced astigmatism [8-10].

In these procedures, meticulous care should always be taken to protect the endothelial cells. Too much manipulation of the endothelial donor lamella may result in a rapid reduction of endothelial cell count or, in a worst case scenario, in the development of primary graft failure. One difficulty during DSAEK/nDSAEK surgery is air management and donor endothelial lamella centering. These maneuvers are especially difficult in small Asian eyes with their shallow anterior chambers [10]. Here, we describe and demonstrate the usefulness of the no-touch technique for donor centering in Asian eyes, as well as the development and successful use of a new donor adjuster for DSAEK.

\section{Surgical Techniques and Clinical Results}

\section{$D S A E K / n D S A E K$}

All DSAEK/nDSAEK procedures were performed as previously described $[5,10]$. In brief, the donor tissue was dissected with a microkeratome (ALTK Cbm; Moria Japan KK, Tokyo, Japan) equipped with a 300- $\mu \mathrm{m}$ head. After microkeratome dissection, the donor tissue was transferred to a punching system and cut with an 8.0-mm diameter punch (Barron donor cornea punch; Katena Products Inc., Denville, N.J., USA). Descemet's membrane was stripped in a circular pattern in DSAEK but neither stripped nor scored in nDSAEK. Then, inferior iridectomy at the 6 o'clock position was created using a 25-gauge vitreous cutter (MIDLAB, San Leandro, Calif., USA). Four venting incisions were created in each quadrant of the cornea in advance for interface fluid drainage. In all cases, the donor endothelial lamella was inserted into the anterior chamber by the pull-through technique using the IOL implantation sheet glide (model GLDE10; Alcon Laboratories, Inc., Fort Worth, Tex., USA) and the Busin glide (catalog No. 19098; Moria, Antony, France; i.e. Kobayashi's double glide technique) [10]. After securing the wound with an interrupted 10-0 nylon suture, air was injected to attach the donor lamella to the posterior stromal surface.

No-Touch Technique for Donor Centering

After air injection into the anterior chamber, donor centering was performed. When the donor dislocated slightly from the center, simple corneal surface massage in the required direction was performed (fig. 1). When the donor dislocated more to the peripheral cornea, ocular tilting in the required direction was performed in addition to corneal massage (fig. 2 ). 


\section{Donor Centering Using the DSAEK Donor Adjuster}

In cases of severe donor dislocation where both no-touch techniques (with or without ocular tilting) failed, the DSAEK donor adjuster (catalog No. AE7806; ASICO, Westmont, Ill., USA,) was used. This device resembles a curved reverse Sinskey hook with a 30 -gauge cannula and allows air injection during donor centering. After inserting this device into the anterior chamber from a side port, the donor was moved centrally by slightly pressing the endothelial side of the donor (fig. 3 ).

\section{Clinical Results}

The retrospective chart review was approved by the Ethical Committee of Kanazawa University Graduate School of Medical Science and followed the tenets of the Declaration of Helsinki. The records were evaluated for 12 patients (3 men, 9 women; age [mean \pm standard deviation (SD)] $75.3 \pm 10.9$ years) with bullous keratopathy who had undergone DSAEK (2 patients) or nDSAEK (10 patients).

The no-touch technique using simple corneal surface massage was successful in 4 cases (33.3\%), while 4 additional cases required ocular tilting (33.3\%) in addition to corneal surface massage. The no-touch technique was ineffective in the remaining 4 cases (33.3\%); however, the DSAEK donor adjuster was used successfully and easily in a single attempt for these patients.

The mean endothelial cell density \pm SD after 6 months was 2,126 \pm 249 cells $/ \mathrm{mm}^{2}$ in the no-touch technique group (with and without ocular tilting, $n=8$ ), representing a $28.3 \%$ mean cell loss from preoperative donor cell measurements, and 1,951 \pm 478 cells $/ \mathrm{mm}^{2}$ in the donor adjuster group $(n=4)$, representing a $31.2 \%$ mean cell loss from preoperative donor cell measurements. Comparing the endothelial cell loss rate between the no-touch technique group and the donor adjuster group, there was no significant difference at 6 months $(\mathrm{p}=0.436$, Mann-Whitney U test; SPSS Statistics version 17.0; SPSS Inc., Chicago, Ill., USA).

\section{Discussion}

When donor dislocation after air injection is slight, simple corneal surface massage is enough for donor centering [3]. This technique is called 'no-touch technique' because there is no direct manipulation of the donor endothelial lamella. In this retrospective review, this technique was successful in 4 cases (33.3\%). When donor dislocation is moderate, simple corneal surface massage is not enough to move the donor centrally; with simple corneal surface massage, the donor sometimes moves in the opposite direction to the far periphery. In such cases, ocular tilting is required in addition to corneal massage. This technique was successful in 4 additional cases (33.3\%).

When both no-touch techniques fail due to severe donor dislocation, another strategy is needed. In such cases, surgeons usually use a reverse Sinskey hook to position the donor; however, sometimes anterior chamber air escapes from the side port, resulting in a flat chamber and donor dislocation. The surgeon is forced to readjust the donor again. If this happens repeatedly, donor endothelial cells may be severely damaged by direct contact with the iris. To circumvent such difficulties as well as to simplify this process, we developed the DSAEK donor adjuster with a blunt tip. It acts as both a reverse Sinskey hook and a 30-gauge air cannula, allowing air injection that maintains anterior chamber depth throughout the procedure. Also, the device is curved for better manipulation in the anterior chamber. Using this device, the surgeon can simultaneously hold the donor in place while injecting the air bubble. In this review, the DSAEK donor adjuster was successful in all 4 cases (33.3\%) where the no-touch technique (with or without ocular tilt) failed. Although this device was specifically developed for DSEAK donor centering, similar devices may have been used by many surgeons with either commercial cystotomes or pre-bent 27- or 30-gauge needles. One problem of such a handmade device is that the sharp tip of the needle 
might stick into the donor stroma, which may result in difficulty for removing the needle from the donor.

In conclusion, the no-touch technique (with or without ocular tilt) is useful for better control of DSAEK donor centering in most cases. The DSAEK donor adjuster shows great promise in selected cases (e.g. those unsuccessfully managed with the no-touch technique).

\section{Acknowledgement}

This study was supported by a Grant-in-Aid for Scientific Research (C) KAKENHI, Japan (No. 22591934).

\section{Disclosure Statement}

None of the authors have a financial or proprietary interest in any material or method mentioned.
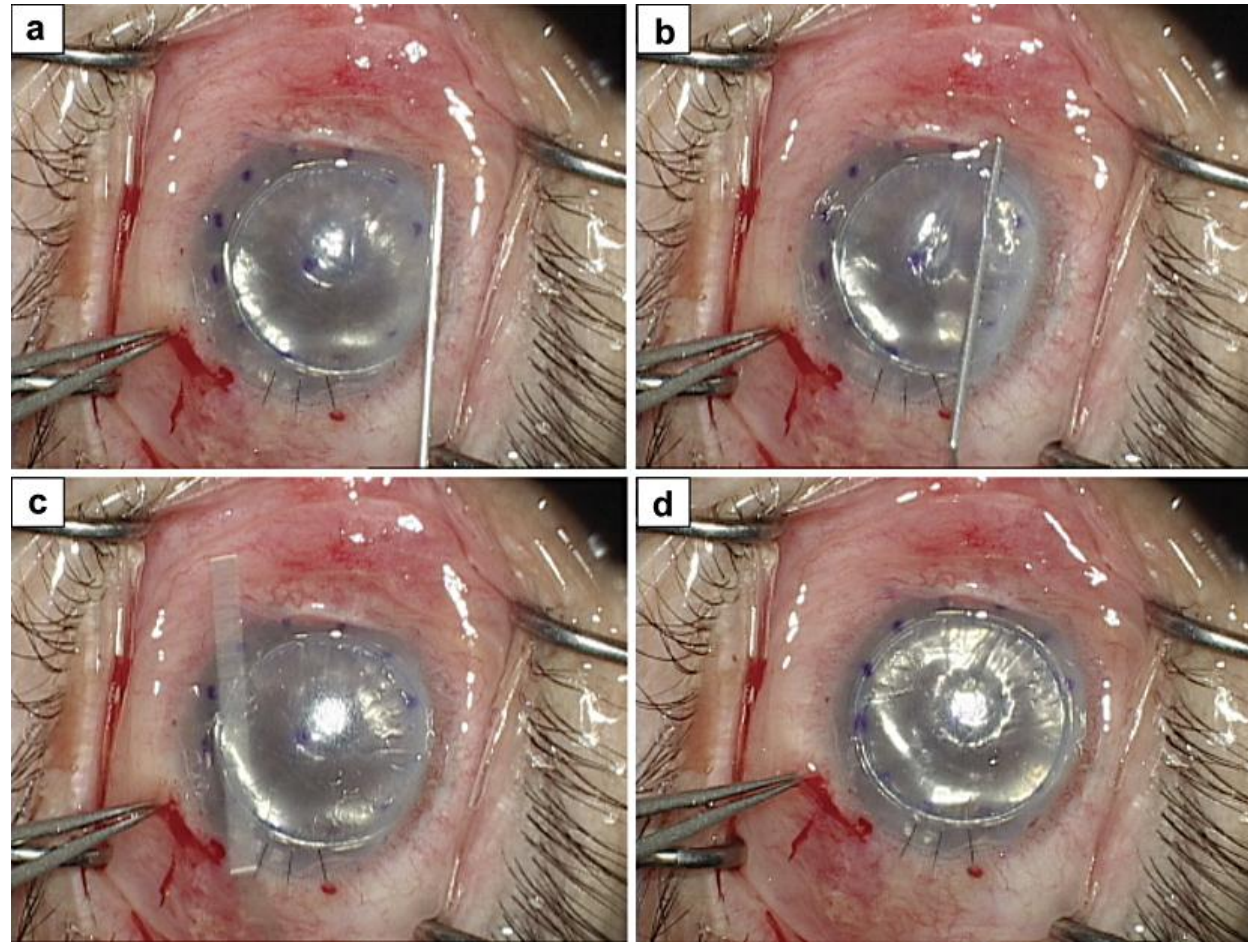

Fig. 1. No-touch technique without ocular tilt. a After air injection into the anterior chamber, donor dislocation is slight. In such cases, simple corneal surface massage is used to direct donor movement. b, c The corneal surface is massaged vigorously from edge to edge using a blunt needle. d After corneal surface massage, the donor is positioned centrally. 

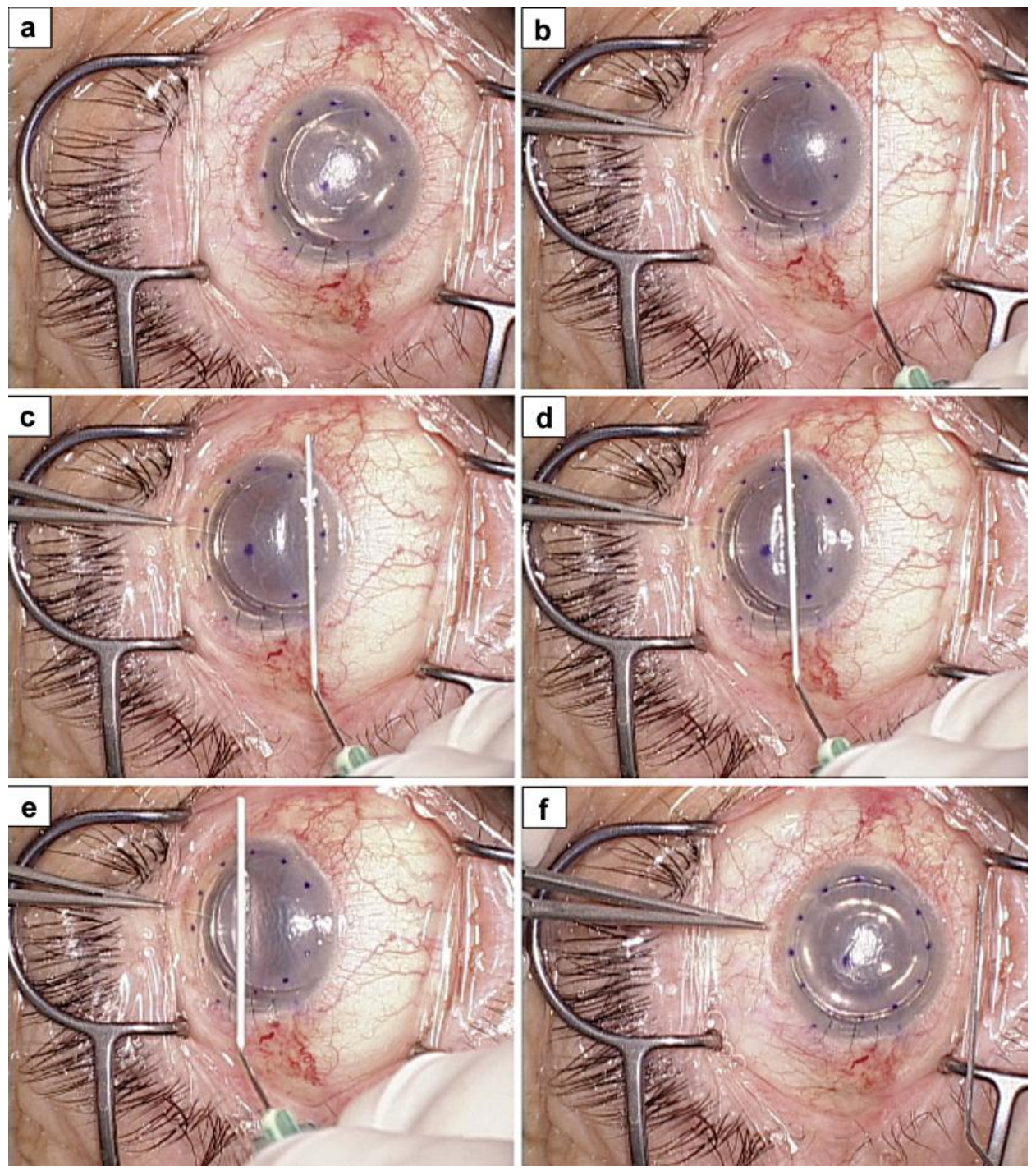

Fig. 2. No-touch technique with ocular tilt. a After air injection into the anterior chamber, donor dislocation is moderate. In such cases, simple corneal surface massage may not succeed. Instead, the donor moves in the opposite direction to the periphery and ocular tilting in the indicated direction is required in addition to corneal surface massage (b-e). f After corneal surface massage with ocular tilt, the donor is positioned centrally. 

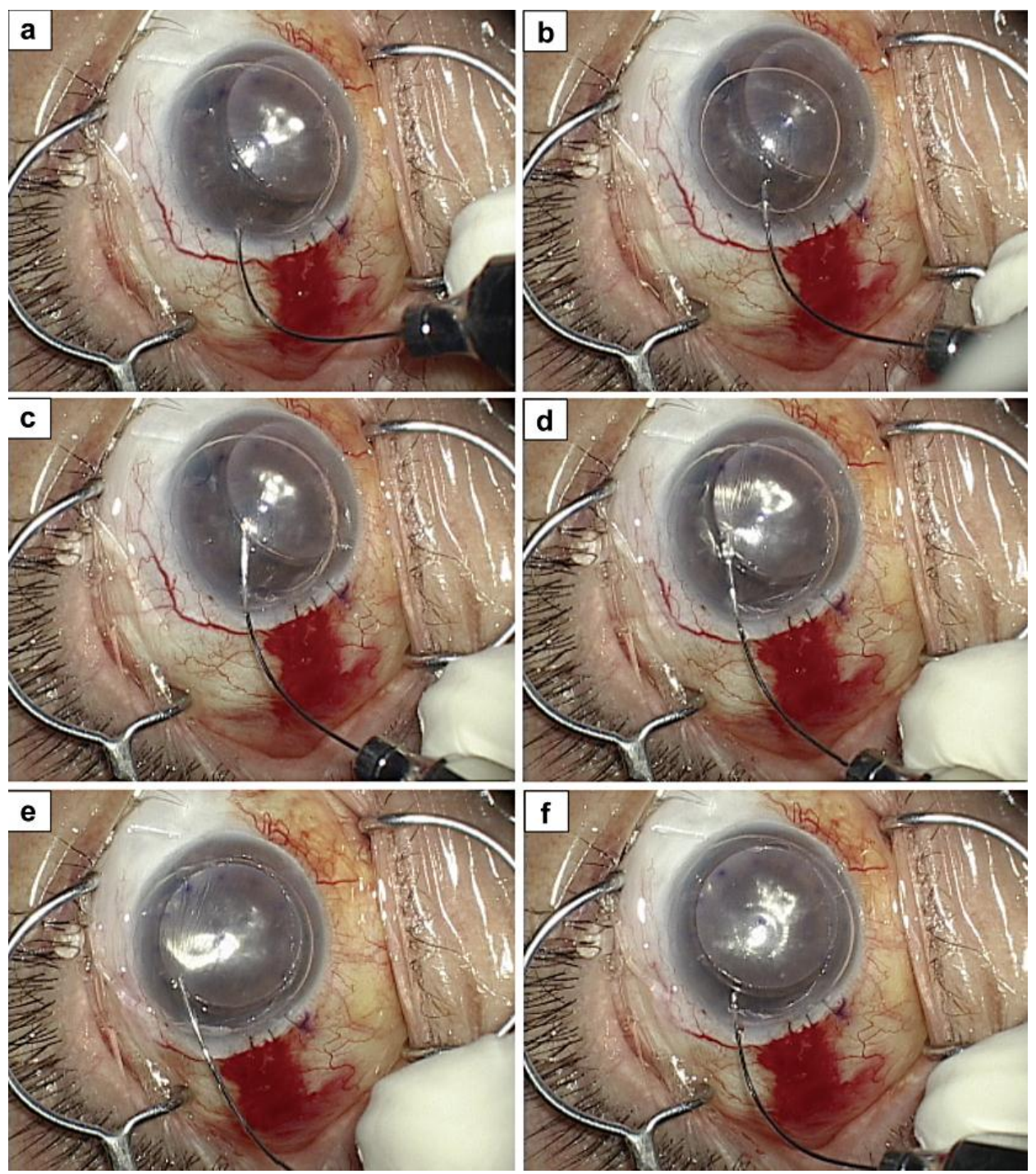

Fig. 3. Donor centering using the DSAEK donor adjuster. a Donor dislocation is severe and the notouch technique is ineffective. $\mathbf{b}$ After inserting the DSAEK donor adjuster into the anterior chamber from a side port, the anterior chamber is collapsed. c Air is injected through the DSAEK donor adjuster. $\mathbf{d}$, e The donor is moved centrally while injecting air into the anterior chamber. $\mathbf{f}$ The donor is positioned centrally, and additional air is injected into the anterior chamber using the DSAEK donor adjuster. 


\section{References}

1 Melles GR, Eggink FA, Lander F, Pels E, Rietveld FJ, Beekhuis WH, Binder PS: A surgical technique for posterior lamellar keratoplasty. Cornea 1998;17:618-626.

-2 Terry MA, Ousley PJ: Deep lamellar endothelial keratoplasty visual acuity, astigmatism, and endothelial survival in a large prospective series. Ophthalmology 2005;112:1541-1548.

-3 Price FW Jr., Price MO: Descemet's stripping with endothelial keratoplasty in 200 eyes: early challenges and techniques to enhance donor adherence. J Cataract Refract Surg 2006;32:411-418.

4 Gorovoy MS: Descemet-stripping automated endothelial keratoplasty. Cornea 2006;25:886-889.

5 Kobayashi A, Yokogawa H, Sugiyama K: Non-Descemet stripping automated endothelial keratoplasty (nDSAEK) for endothelial dysfunction secondary to argon laser iridotomy. Am J Ophthalmol 2008;146:543-549.

6 Price MO, Price FW Jr., Trespalacios R: Endothelial keratoplasty technique for aniridic aphakic eyes. J Cataract Refract Surg 2007;33:376-379.

7 Price FW Jr., Price MO: Endothelial keratoplasty to restore clarity to a failed penetrating graft. Cornea 2006;25:895-899.

$>8$ Kobayashi A, Yokogawa H, Sugiyama K: In vivo laser confocal microscopy after non-Descemet stripping automated endothelial keratoplasty. Ophthalmology 2009;116:1306-1313.

-9 Kobayashi A, Mawatari Y, Yokogawa H, Sugiyama K: In vivo laser confocal microscopy after Descemet's stripping with automated endothelial keratoplasty. Am J Ophthalmol 2008;145:977-985.

10 Kobayashi A, Yokogawa H, Sugiyama K: Descemet's stripping with automated endothelial keratoplasty for bullous keratopathies secondary to argon laser iridotomy-preliminary results and usefulness of double-glide donor insertion technique. Cornea 2008;27(suppl 1):S62-S69. 